Fundación

Miguel Lillo

Tucumán

Argentina

\title{
Morfología larval y datos biológicos de Leucochrysa (Nodita) cruentata (Neuroptera: Chrysopidae), primer registro en Argentina
}

\author{
Larval morphology and biology features of Leucochrysa (Nodita) \\ cruentata (Neuroptera: Chrysopidae), including its first record \\ from Argentina
}

\footnotetext{
Mariángela Margagliotti 1,2; Marcela Margagliotti Reguilón 1,2; Carmen Reguilón ${ }^{1}$

1 Fundación Miguel Lillo, Instituto de Entomología. Miguel Lillo 251. (4000) San Miguel de Tucumán. Corresponding author: creguilon@lillo.org.ar

2 Facultad de Ciencias Naturales e IML, UNT. Miguel Lillo 205. (4000) San Miguel de Tucumán.
}

\section{RESUMEN}

Leucochrysa es el género más grande y diverso de la familia Chrysopidae, con aproximadamente 190 especies descritas. Se distribuye desde el norte de los Estados Unidos al norte, hasta el noroeste de Argentina en el sur, presentando la mayor diversidad de especies en la región Neotropical. Los dos subgéneros: L. (Leucochrysa) McLachlan y $L$. (Nodita) Navás, están representados en la Argentina por cinco y dos especies, respectivamente. Leucochrysa (Nodita) cruentata se ha encontrado por primera vez en el noroeste de Argentina en la zona de pedemonte de las Yungas, en agroecosistemas de Citrus y otros cultivos colindantes a la misma, lo cual presupone que puede ejercer un importante papel en el control biológico de plagas, más aun teniendo en cuenta que es activa depredadora en estado larval de pequeños fitófagos. La biología de las especies neotropicales de Nodita, así como su morfología larval, son poco conocidas. En este trabajo registramos una tercera especie en Argentina de L. (Nodita): Leucochrysa (Nodita) cruentata (Schneider), se presentan las primeras descripciones de la morfología externa de la larva de tercer estadio, además de ilustraciones, detalles de la quetotaxia y datos de su ciclo biológico. Se aporta una clave para la identificación del tercer estadio larval de las seis especies neotropicales de Leucochrysa (Nodita) de las que se conocen sus larvas.

Palabras clave — Leucochrysini, larva, ciclo biológico, Sudamérica.

Ref. bibliográfica: Margagliotti, M.; Margagliotti Reguilón, M.; Reguilón, C. 2021. "Morfología larval y datos biológicos de Leucochrysa (Nodita) cruentata (Neuroptera: Chrysopidae), primer registro en Argentina". Acta zoológica lilloana 65 (2): 230-240. doi: https://doi.org/10.30550/j.azl/2021.65.2/2021-09-15

> Recibido: 11 de junio 2021 - Aceptado: 15 de septiembre 2021.

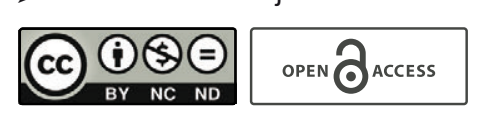

- URL de la revista: http://actazoologica.lillo.org.ar

- Esta obra está bajo una Licencia Creative Commons Atribución - No Comercial - Sin Obra Derivada 4.0 Internacional. 


\section{ABSTRACT}

Leucochrysa is the largest and most diverse genus of Chrysopidae. It includes approximately 190 species. It is distributed from northern United States in the north, to northwestern Argentina in the south, with the greatest species diversity in the Neotropical region. The two subgenera: L. (Leucochrysa) McLachlan and L. (Nodita) Navás previously were represented in Argentina by five and two species respectively Here we report a third Argentinian species of $L$. (Nodita): Leucochrysa (Nodita) cruentata (Schneider) from the Yungas piedmont zone of northwestern Argentina. Adults were collected in Citrus and adjacent agroecosystems. The species' habitat association and active, predaceous larvae lead us to propose that $L$. (N.) cruentata might perform an important role in the biological control of insect pests. The neotropical species of Nodita, including their biological features and their larval morphology, are poorly known. Here, we report the first record of $L$. (N.) cruentata in Argentina. We also describe for the first time the external morphology of the species' third larval stage; we include illustrations, details of the chaetotaxy, and life cycle data. In addition, we provide a key for identifying the third instars of the six neotropical Leucochrysa (Nodita) species for which larvae are known.

Palabras clave - Leucochrysini, larvae, biological cycle, South America.

\section{INTRODUCCIÓN}

El género Leucochrysa McLachlan es el más grande y diverso de la familia Chrysopidae (Mantoanelli y Albuquerque, 2007), con aproximadamente 190 especies descriptas (Adams y Penny, 1987; Brooks y Barnard, 1990; Freitas y Penny, 2001). Se distribuye desde el norte de los Estados Unidos al norte, hasta el noroeste de Argentina en el sur (Penny, 1978), presentando la mayor diversidad de especies en la región Neotropical (Oswald y Machado, 2018). Se conocen siete especies de Estados Unidos (Tauber, 2004) y el resto son neotropicales (Penny, Adams, Stange, 1997; Freitas y Penny, 2001; Freitas, 2005; Mantoanelli, Tauber, C., Albuquerque, Tauber, M., 2011; Tauber, C., Albuquerque, Tauber, M., 2008; Tauber et al., 2011; Tauber, C., Sosa, Albuquerque, Tauber, M., 2013).

Los dos subgéneros: L. (Leucochrysa) McLachlan y L. (Nodita) Navás, están representados en la Argentina por cinco y dos especies, respectivamente (González Olazo y Reguilón, 2008).

Leucochrysa (Nodita) cruentata (Schneider, 1851) se encontró por primera vez en el noroeste de Argentina en la ecorregión de Yungas, lo interesante es su frecuente presencia en agroecosistemas de Citrus y otros cultivos colindantes a la zona pedemontana de Tucumán, lo cual presupone que puede ejercer un importante papel en el control biológico de plagas, más aun teniendo en cuenta que es activa depredadora en estado larval de pequeños fitófagos (Freitas y Penny, 2001; De Castro Coitinho, de Lacerda, dos Santos, da Silva, 2018; Costa et al., 2020).

La morfología del adulto, cabeza, tórax, extremo abdominal, venación alar y genitalia ha sido ampliamente descrita e ilustrada por Freitas y Penny (2001), lo cual hace posible su identificación. 
Se ha descrito e ilustrado la morfología larval de cuatro especies de Nodita presentes en los Estados Unidos (Tauber, 2004). Hasta el presente el conocimiento de la biología de las especies neotropicales del subgénero Nodita, se limita a cuatro especies presentes en Brasil (Mantoanelli et al., 2011) y a Leucochrysa (Nodita) vignisi de Brasil y Argentina (Reguilón y Heredia, 2011).

En este trabajo se cita por primera vez a Leucochrysa (N.) cruentata para Argentina, se presenta, en forma detallada, la morfología externa de la larva del tercer estadio a través de descripciones, ilustraciones y cuadro de quetotaxia. Se incluyen imágenes de la prepupa y pupa y se aportan datos de su distribución, ciclo biológico y una clave para la identificación del tercer estadio larval de las seis especies neotropicales de Leucochrysa (Nodita) de las que se conocen sus larvas.

\section{MATERIALES Y MÉTODOS}

Los ejemplares adultos fueron recolectados en marzo del año 2018 en vegetación natural de la zona pedemontana de Yungas colindante a cultivos cítricos del departamento de Tafí Viejo y en cultivos de Citrus y soja del departamento Famaillá, Tucumán, Argentina. La captura de los insectos adultos se realizó utilizando red entomológica, aspiradores de boca y trampa de luz.

El estudio sobre la morfobiología de $L$. (N.) cruentata, se llevó a cabo en el Laboratorio de Cría de Biocontroladores (LACRIBIO) del Instituto de Entomología de la Fundación Miguel Lillo, Tucumán, Argentina. Para establecer la cría, a fin de caracterizar la morfología larval y datos sobre su ciclo biológico, se partió de una cohorte de 33 huevos que fueron mantenidos en cámara de cría a una temperatura de 20 a $21^{\circ} \mathrm{C}$ y humedad relativa del $60 \%$. La nutrición de los adultos fue asegurada con una dieta a base de miel, levadura de cerveza inactiva, polen, agua en proporción 2:1:1:2 y suplementada con germen de trigo y vitaminas, modificada de RamírezDelgado, López-Arroyo, González-Hernández, Badii-Zabeh (2007) y Solano, Guillén, Conejo (2019). Las larvas fueron alimentadas con huevos de Sitotroga cerealella Olivier (Lepidoptera: Gelechiidae).

Para la descripción e ilustración de los caracteres morfológicos del tercer estadio de $L$. (N.) cruentata se observaron las larvas, luego que fueron fijadas con solución KAAD y posteriormente conservadas en alcohol al $65 \%$ glicerinado (Stehr 1987). Para el detalle de la quetotaxia se siguió a Tauber et al. (2011), Tauber (2004), Mantoanelli, Albuquerque, Tauber, C., Tauber, M. (2006) y Reguilón (2010, 2011). Las ilustraciones fueron realizadas con el uso de un microscopio binocular con cámara clara. Para la determinación de los adultos se utilizó la clave de Freitas y Penny (2001).

Los ejemplares identificados (tanto adultos como inmaduros) se depositaron en la Colección Entomológica Instituto-Fundación Miguel Lillo (IFML). 

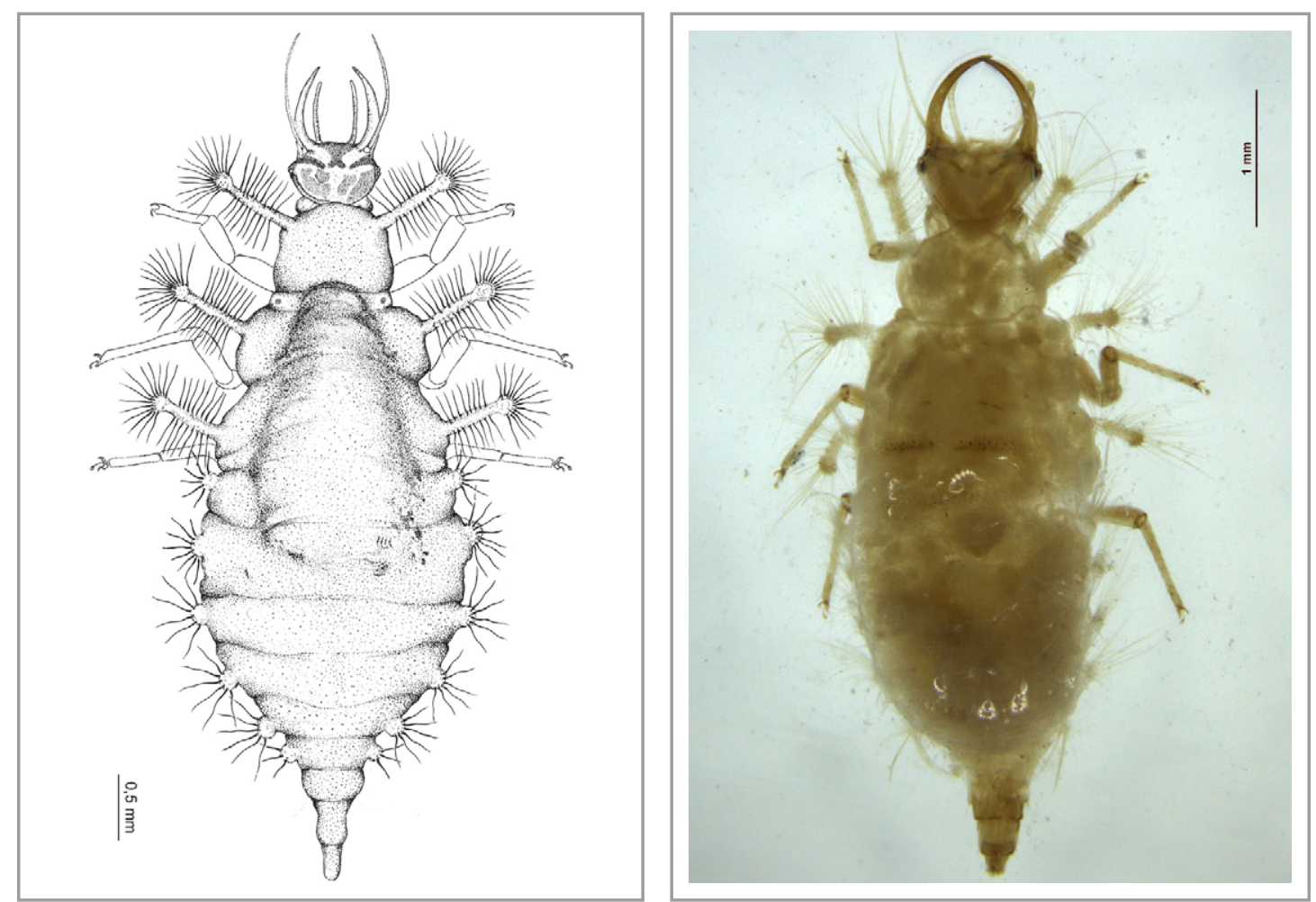

Fig. 1. Leucochrysa (Nodita) cruentata: (a) dibujo y (b) fotografía vista dorsal de la larva de tercer estadio.

Fig. 1. Leucochrysa (Nodita) cruentata: (a) illustration and (b) photography dorsal view of the third larval instar.

\section{RESULTADOS \\ Descripción del tercer estadio larval de Leucochrysa (Nodita) cruentata}

Cuerpo (Fig. 1).— Fusiforme, castaño claro, más ancho en las regiones del mesotórax, metatórax y los cinco primeros segmentos abdominales, adelgazándose gradualmente a partir del sexto segmento hacia el extremo anal. Larvas portadoras de residuos sobre el dorso, cuerpo cubierto de setas cortas y largas y un par de tubérculos setígeros laterales largos y prominentes (ocho veces más largos que anchos), en cada segmento del tórax, cortos y subesféricos en el abdomen.

Cabeza (Fig. 2).- Más bien grande, más ancha a nivel de los ojos, con la base que se estrecha para articularse con el primer segmento del tórax, de color castaño claro, con marcas cefálicas intermandibular (im) unida a la frontal (fr), marca posterior o postfrontal (post) y marcas epicraneales, media (epi-m) y lateral (epi-1) evidentes, castañas rojizas. Mandíbulas y maxilas aproximadamente 1/4 más largas que el largo de la cabeza, distalmente aguzadas, castañas oscuras; antenas ámbar claro, 2/4 más largas que las mandíbulas, escapo corto y grueso, de pigmentación castaña, pedicelo corto y ligeramente más estrecho, flagelo largo, con bandas transversales de engrosamientos que dan aspecto anillado. Palpos labiales trisegmentados, castaños claros, de longitud 1/4 más cortos que las mandíbulas. 

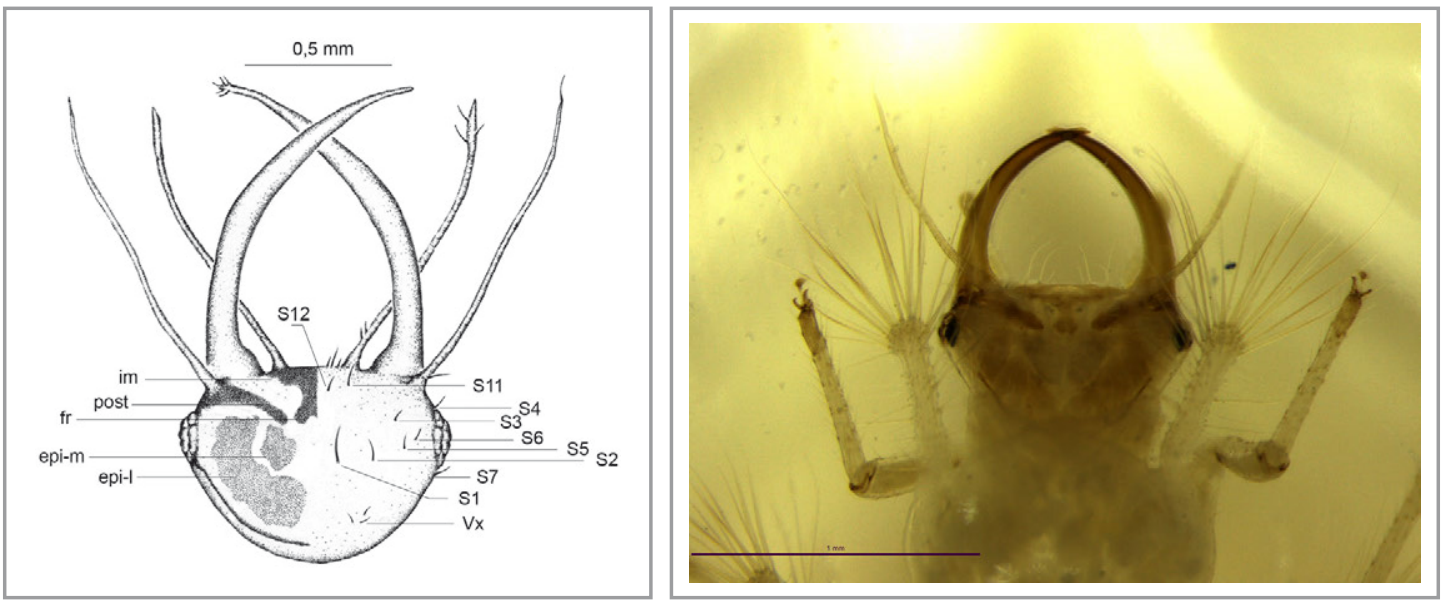

Fig. 2. Leucochrysa (N.) cruentata (tercer estadio larval): a) dibujo de la cabeza con los detalles de las marcas cefálicas: im (intermandibular); fr (frontal); post (postfrontal); epi-m (epicraneal media) y epi-I (epicraneal lateral); Vx (tres setas cortas alrededor del poro). Setación cefálica primaria: setas S1-S12. b) fotografía del aspecto y coloración de la cabeza, detalles de las marcas cefálicas y tubérculos setígeros protorácicos.

Fig. 2. Leucochrysa (N.) cruentata third larval instar: a) illustration dorsal head markings: im (intermandibular); fr (frontal); post (postfrontal); epi-m (mesal section of the epicranial) y epi-I (lateral section of the epicranial); Vx (three short posterior setae surrounding a pore). Primary cephalic setae 1-12: S1-S12. b) photography dorsal head, details cephalic markings and prothoracic tubercle setigerous.

Tórax (Fig. 3).— Protórax con escleritos Sc1 y Sc2 no evidentes; setas del protórax pareadas, cortas S1-S5 y R1 (fila de tres setas anteriores pequeñas); mesotórax y metatórax sombreados de castaño claro, con dos hileras dobles de setas dorsales cortas, superiores e inferiores. Largo de los Tubérculos setígeros aproximadamente 3/4 del ancho del protórax con, setas laterales y apicales (SL y Sa) largas, en número de 17,8 $\pm 1,8$ y $14,1 \pm 0,9$ respectivamente por tubérculo.

Abdomen (Fig. 4). - Castaño claro del primer segmento al séptimo, el resto de los segmentos castaños oscuros. Se distingue una marca de contorno definido, suboval, castaño rojiza en el dorso de la larva del tercer estadio que se define en este trabajo con el nombre de "marca dorsal" (Md), la que se extiende desde el metatórax hasta el tercer segmento del abdomen.

En el primer segmento abdominal se presenta una hilera de setas dorsomediales superiores, en el segundo segmento se observa una hilera de setas dorsomediales inferiores. En los segmentos 3 a 6 se presentan dos hileras de setas dorsomediales cortas, de posición superior e inferior en cada segmento. Tubérculos setígeros laterales prominentes, subesféricos, que presentan de seis a ocho setas apicales. Se destaca la presencia de una seta larga dorsolateral media a cada lado del octavo segmento abdominal, de longitud de 3/4 del ancho del mencionado segmento y que damos en llamar SL8, y la presencia de setas cortas, cuatro dorsomediales superiores, seis dorsomediales inferiores y 3 laterales a cada lado del mencionado segmento. El noveno segmento abdominal presenta dos setas cortas dorsomediales superiores, dos dorsomediales inferiores y dos laterales a cada lado del segmento. 


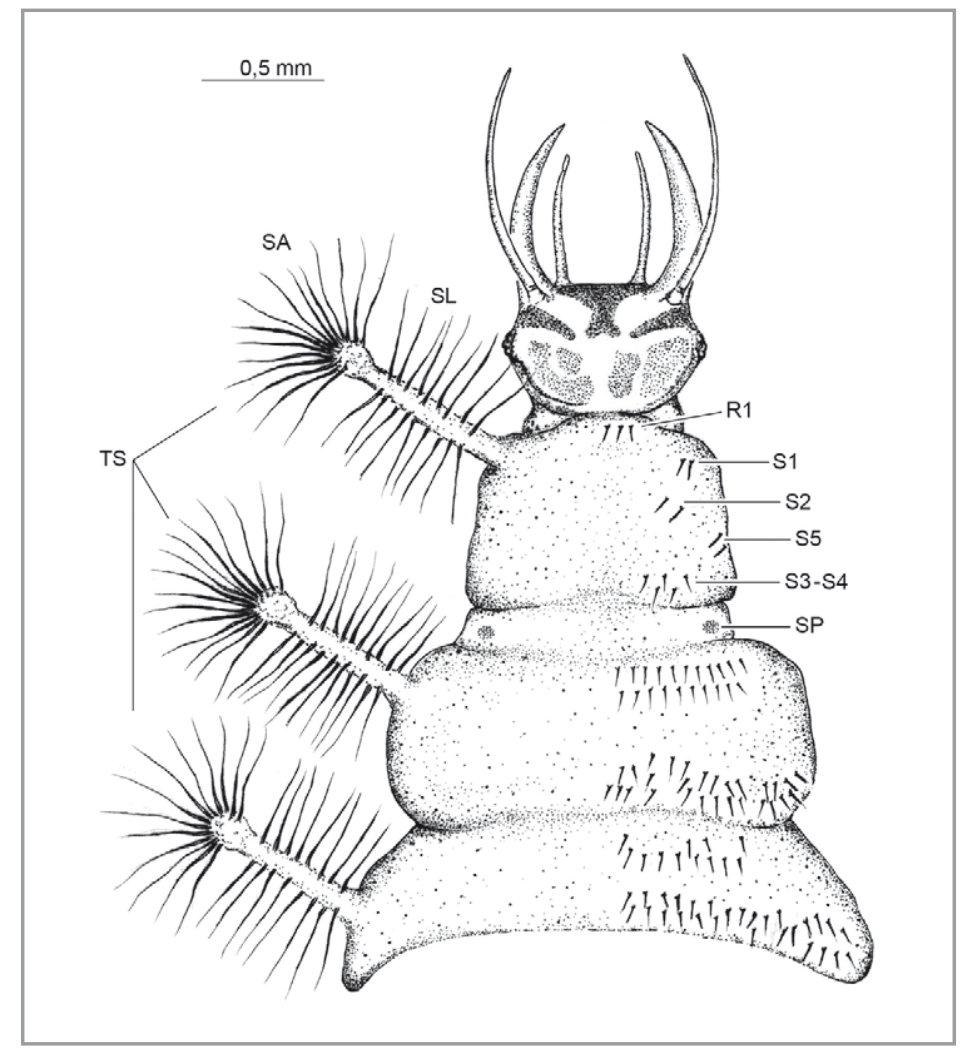

Fig. 3. Leucochrysa (N.) cruentata (tercer estadio larval): vista dorsal del tórax. Mitad derecha, detalle de la setación torácica: R1, fila de tres pequeñas setas anteriores; S1-S5, setas torácicas primarias; Sp, espiráculo. Mitad izquierda, detalle de la setación de los TS, tubérculos setígeros: SL, setas laterales del tubérculo; SA, setas apicales del tubérculo.

Fig. 3. Leucochrysa (N.) cruentata third larval instar: Thorax, dorsal. Right side, detail of thoracic setae: R1, row of three small anterior setae; S1-S5, primary thoracic setae; Sp, spiracle. Left side, detail of tubercle setigerous TS: SL, setae on lateral tubercle; SA, setae on apical tubercle.

Longitud promedio del cuerpo 6,91 a 7,12 $\mathrm{mm}$; ancho de la cabeza 0,94 a 0,98 $\mathrm{mm}$; ancho del tórax a la altura del metatórax 1,91 a $1,94 \mathrm{~mm}$; longitud de las mandíbulas 0,89 a $0,95 \mathrm{~mm}$; longitud de los tubérculos setígeros toráxicos 0,55 a $0,65 \mathrm{~mm}$.

El detalle de la quetotaxia torácica y abdominal del tercer estadio larval de $L$. $(N$.$) cruentata se presenta en las Tablas I y II.$

\section{Datos biológicos}

Los datos obtenidos del ciclo de vida de $L$. (N.) cruentata se presentan en la Tabla III, donde se puede observar que en condiciones controladas de temperatura y humedad relativa $\left(20-21^{\circ} \mathrm{C}\right.$ y $65 \%$, respectivamente), el ciclo de vida (huevo a emergencia del adulto) se desarrolla en aproximadamente 36 días. El promedio de oviposición por hembras es de 33,8 $\pm 1,89$ huevos y el porcentaje de supervivencia en las condiciones establecidas y con la alimentación usada en este estudio fue de un $70 \%$. Se presenta el aspecto general de la prepupa y pupa en la Fig. 5. 


\section{DISCUSIÓN}

En este trabajo se cita por primera vez para la Argentina a L. (N.) cruentata asociada a la ecorregión de Yungas y a cultivos colindantes a la misma. Se presenta la morfología del tercer estadio larval, se detalla la quetotaxia torácica y abdominal, se presentan datos sobre su ciclo biológico, lo que constituye el primer aporte al conocimiento de la biología de esta especie de Leucochrysa subgénero Nodita presente en la región Neotropical. Se habían descrito hasta el presente, en forma detallada, la morfología larval de especies del subgénero Nodita de la región Holártica (Tauber,

Tabla I. Detalle de la quetotaxia torácica del tercer estadio larval de Leucochrysa (N.) cruentata. C $=$ Setas cortas; $\mathrm{L}=$ Setas largas (relación de longitud L: $C=4: 1$ ); $0=$ Setas ausentes.

Tabla I. Detail of the thoracic chaetotaxy of the third larval instar of Leucochrysa (N.) cruentata. C $=$ short setae; $\mathrm{L}=$ long setae (ratio $\mathrm{L}: \mathrm{C}=4: 1$ ); $0=$ absent setae.

\begin{tabular}{|c|c|c|c|c|}
\hline \multirow{7}{*}{ 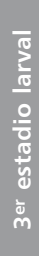 } & Grupos de setas & Protórax & Mesotórax & Metatórax \\
\hline & Dorsomediales sup. & $6 C$ & $42 \pm 2 C$ (en dos filas) & $24 \pm 2 C$ (en dos filas) \\
\hline & Dorsomediales inf. & $10 \mathrm{C}$ & $40 \pm 2 C$ (en tres filas) & $38 \pm 2 C$ (en dos filas) \\
\hline & Laterales sup. & $8 \mathrm{C}$ & 0 & 0 \\
\hline & Laterales inf. & $4 C$ & $16 \pm 2 C$ (en dos filas) & $18 \pm 2 C$ (en dos filas) \\
\hline & Tubérculos: Setas Laterales & $18 \pm 2 L$ & $21 \pm 1 \mathrm{~L}$ & $16 \pm 2 L$ \\
\hline & Tubérculos: Setas apicales & $15 \pm 1 L$ & $15 \pm 1 L$ & $13 \pm 1 L$ \\
\hline
\end{tabular}

Tabla II. Detalle de la quetotaxia abdominal del tercer estadio larval de Leucochrysa (N.) cruentata. C $=$ Setas cortas; $\mathrm{L}=$ Setas largas (relación de longitud L: $\mathrm{C}=4: 1$ ); SL8 = Setas largas del segmento 8; $0=$ Setas ausentes.

Tabla II. Detail of the abdominal chaetotaxy of the third larval instar of Leucochrysa (N.) cruentata. $C=$ short setae; $\mathrm{L}=$ long setae (ratio $\mathrm{L}: \mathrm{C}=4: 1$ ); $\mathrm{SL8}=$ long setae on eight abdominal segment; $0=$ absent setae.

\begin{tabular}{|c|c|c|c|c|c|c|c|c|c|c|c|}
\hline \multirow{3}{*}{$\bar{c}$} & \multirow{2}{*}{ Grupos de setas } & \multicolumn{10}{|c|}{ Segmentos abdominales } \\
\hline & & $1^{\circ}$ & $2^{\circ}$ & $3^{\circ}$ & $4^{\circ}$ & $5^{\circ}$ & $6^{\circ}$ & $7^{\circ}$ & $8^{\circ}$ & $9^{\circ}$ & $10^{\circ}$ \\
\hline & Dorsomediales sup. & $24 \pm 2 C$ & 0 & $20 \pm 2 C$ & $28 \pm 2 C$ & $24 \pm 2 L$ & $32 \pm 2 L$ & 0 & $4 C$ & $2 C$ & 0 \\
\hline$\frac{10}{0}$ & Dorsomediales medias & 0 & 0 & 0 & 0 & 0 & 0 & $10 \mathrm{C}$ & 0 & 0 & 0 \\
\hline 응 & Dorsomediales inf. & 0 & $28 \pm 2 C$ & $22 \pm 2 C$ & $40 \pm 2 C$ & $52 \pm 2 C$ & $24 \pm 2 C$ & $8 \mathrm{C}$ & $6 C$ & $2 C$ & 0 \\
\hline 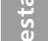 & Laterales sup. & 0 & 0 & 0 & $14 \pm 2 C$ & 0 & 0 & 0 & $2 S L 8$ & 0 & 0 \\
\hline$\stackrel{0}{\infty}$ & Laterales inf. & 0 & 0 & 0 & 0 & 0 & 0 & 0 & $6 C$ & $4 C$ & 0 \\
\hline & $\begin{array}{l}\text { Tubérculo Setígero/ } \\
\text { abdominal }\end{array}$ & 0 & $6 \pm 2 C$ & $8 \pm 2 C$ & $6 \pm 2 \mathrm{~L}$ & $6 \pm 2 L$ & $6 \pm 2 L$ & $3 C$ & $2 \mathrm{C}-1 \mathrm{~L}$ & 0 & 0 \\
\hline
\end{tabular}

Tabla III. Datos del Ciclo Biológico de Leucochrysa (Nodita) cruentata (en condiciones controladas de laboratorio).

Tabla III. Life cycle data of Leucochrysa (Nodita) cruentata (under laboratory controlled conditions).

\begin{tabular}{l|c|c|c}
\hline Estado de desarrollo & N & Duración en días (*) & \% Supervivencia \\
\hline Huevo & 33 & $4,87 \pm 0,69$ & 100 \\
\hline Larva I & 29 & $4,89 \pm 0,72$ & 87,87 \\
\hline Larva II & 29 & $5,51 \pm 0,63$ & 87,87 \\
\hline Larva III & 29 & $4,27 \pm 0,45$ & 87,87 \\
\hline Prepupa & 26 & $2,07 \pm 0,48$ & 78,78 \\
\hline Pupa & 26 & $14,34 \pm 0,62$ & 78,78 \\
\hline Adulto & 23 & $12,43 \pm 0,66$ & 69,69 \\
\hline
\end{tabular}



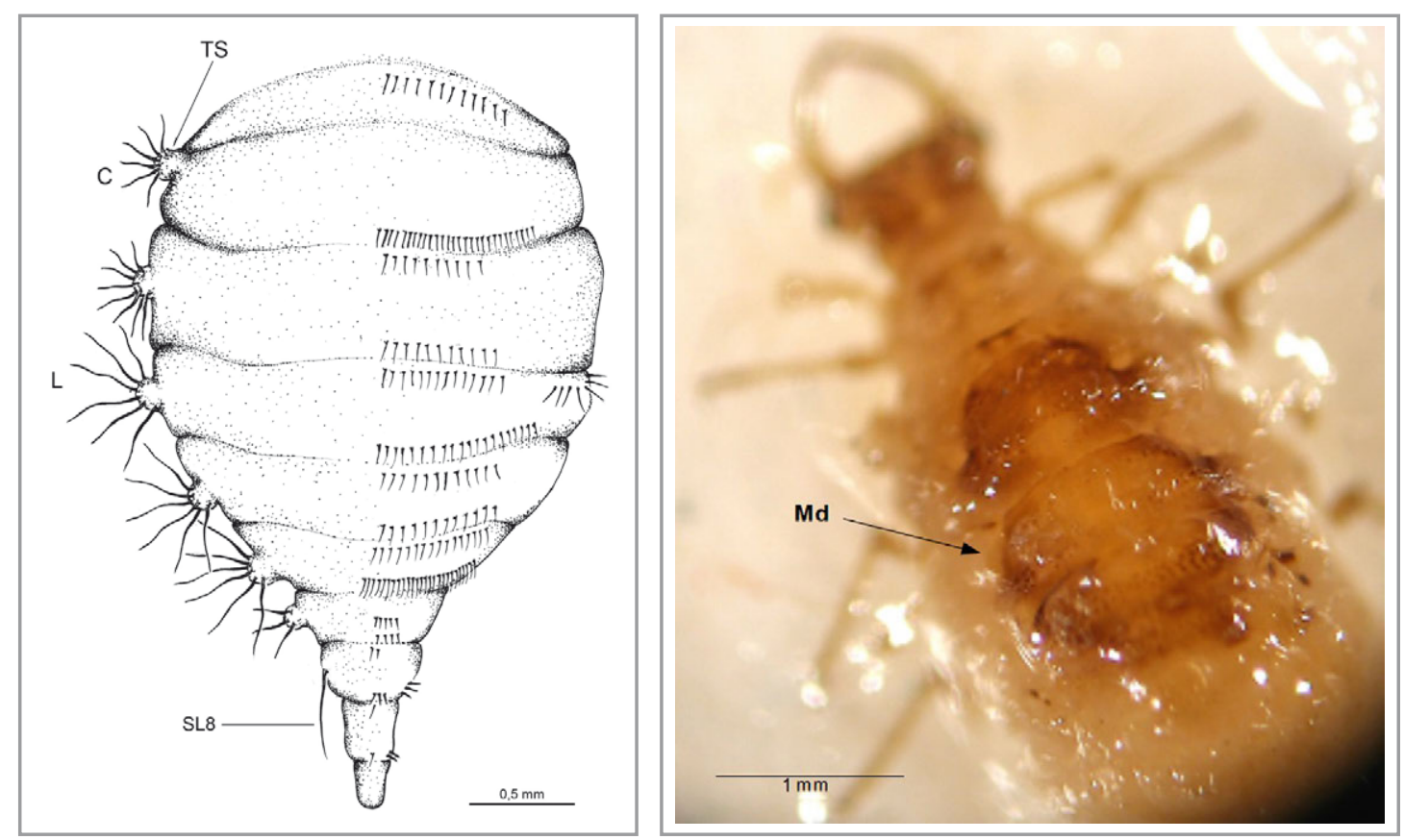

Fig. 4. Leucochrysa (N.) cruentata (tercer estadio larval): vista dorsal del abdomen. a) Dibujo: mitad derecha, detalle de la setación abdominal y mitad izquierda de los TS, tubérculos setígeros. Setas: L, largas; C, cortas; SL8, seta larga dorsolateral media del segmento ocho. b) fotografía: detalle de la Marca dorsal (Md), coloración castaño rojiza.

Fig. 4. Leucochrysa (N.) cruentata third larval instar: abdominal segments dorsal. a) illustration: Right side, detail of abdominal setae and left side, detail of tubercle setigerous, TS. Setae: L, long; C, short; SL8, long setae mesal dorsolateral on eight abdominal segment. b) photography: detail of dorsal marking $(\mathbf{M d})$, reddish brown color.

a)
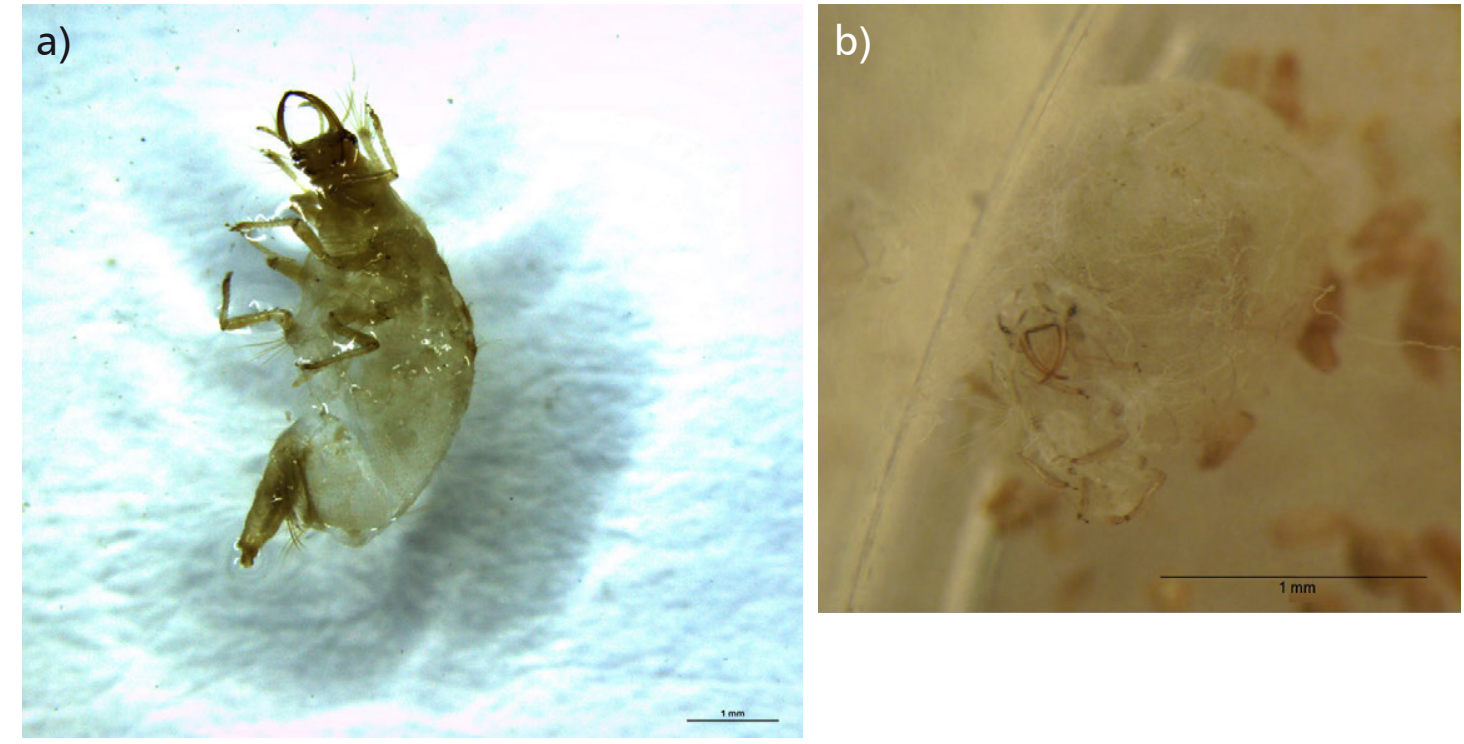

Fig. 5. Leucochrysa (Nodita) cruentata: fotografías de: (a) prepupa, vista lateral y b) pupa.

Fig. 5. Leucochrysa (N.) cruentata: photography: (a) prepupa, lateral view and (b) pupa. 
2004) y de la región neotropical, de Brasil, cuatro especies (Mantoanelli et al., 2011), de Brasil y Argentina L. (N.) vignisi (Reguilón y Heredia, 2011). El presente estudio muestra claramente las diferencias morfológicas entre la larva de tercer estadio de L. (N.) cruentata y de otras especies de Nodita descritas para las regiones Holártica y Neotropical, observando caracteres como las marcas cefálicas, marcas torácicas y setación del cuerpo. Particularmente es importante señalar la estructura observada por primera vez, llamada en este trabajo, Marca dorsal, (Md) en la larva de tercer estadio del género, como así también la seta larga dorsolateral del segmento abdominal 8: SL8. Asimismo, teniendo en cuenta la clave para larvas de cuatro especies de Nodita de Brasil presentada por Mantoanelli et al 2011, e incorporando las dos especies descritas de Argentina, se presenta una nueva clave para la identificación de larvas del tercer estadio del subgénero Nodita del Neotropico.

En cuanto a los datos biológicos podemos indicar que bajo las mismas condiciones de cría en laboratorio (temperatura y humedad relativa) del estudio realizado de la otra especie descrita para Argentina: L. (N.) vignisi, (Reguilón, Heredia, 2011), se obtuvo para $L$. (N.) cruentata, una menor duración del ciclo de vida, mayor promedio de oviposición por hembras y un porcentaje de supervivencia mayor.

Finalmente, el hecho de haber encontrado a la especie no solo en vegetación natural, sino también en agroecosistemas de Citrus y soja en Tucumán, y teniendo en cuenta otros trabajos que indican que un gran número de especies de Leucochrysa (Nodita) se encuentran en cultivos agrícolas (naranja, caucho, anacardo, maíz, guayaba) de Brasil (Freitas, Penny, 2001; Mantoanelli, et al., 2011) y otras especies del género se han citado como activas depredadoras en estado larval de pequeños fitófagos (Costas et al., 2011; de Castro Coitinho, et al., 2018; Tauber, et al., 2008), podemos indicar que $L$. $(N$.) cruentata tiene características que pueden convertirla en un enemigo natural promisorio para su uso en proyectos de control biológico de insectos plagas.

\section{AGRADECIMIENTOS}

Agradecemos al Lic. Hugo Pablo Pereyra, por la realización de las ilustraciones; a la Dra. María Alejandra Molina por las fotografías. Asimismo, deseamos agradecer a los editores y revisores por sus oportunas sugerencias.

El permiso de colecta fue otorgado por la Dirección de Flora, Fauna Silvestre y Suelos de la Provincia de Tucumán, para el Proyecto Z-0040-1 de la Fundación Miguel Lillo, Resol. N ${ }^{\circ}$ 68-2020, Exp. N $^{\circ}$ 2033-330-2019.

\section{FINANCIAMIENTO}

El presente trabajo fue financiado por los Proyectos: Z-0040-1 del Instituto de Entomología, de la Fundación Miguel Lillo y Z-0042-13, FITR - BID No 2437/OC-AR, Banco Interamericano de Desarrollo, Ministerio de Ciencia, Tecnología e Innovación Productiva de la Nación Argentina. 


\section{PARTICIPACIÓN}

La autora de correspondencia planificó, diseño el estudio y participó de la determinación taxonómica, caracterización morfológica y redacción del manuscrito. Las otras dos autoras contribuyeron por igual con los muestreos, acondicionamiento y fijación del material, cría en laboratorio, caracterización morfológica, datos biológicos del ciclo de vida y redacción del manuscrito.

\section{CONFLICTOS DE INTERÉS}

En el presente trabajo no existen conflictos de interés entre los autores o con terceros.

\section{LITERATURA CITADA}

Adams, P. A., Penny, N. D. (1987). Neuroptera of the Amazon Basin. Part 1 1a. Introducción and Chrysopini. Acta Amazónica, 15, 413-479.

Brooks, S. J., Barnard, P. C. (1990). The green lacewings of the world: a generic review (Neuroptera: Chrysopidae). Bulletin of the British Museum (Natural History), Entomology Series, 59, 117-286.

Costa, S. S., Broglio, S. M., Dias-Pini, N. S., Santos, D. S., Santos, J. M., Duque, F. J., Saraiva, W. V. (2020). Developmental biology and functional responses of Leucochrysa (Nodita) azevedoi fed with different prey. Biocontrol Science and Technology, 30, 42-50.

De Castro Coitinho, R. L. B., de Lacerda, C. A., dos Santos, V. F., da Silva, D. M. P. (2018). Predação de ninfas Diaspis echinocacti (Bouché) (Hemiptera: Diaspididae) por larvas de Leucochrysa (Nodita) sp. (Neuroptera: Chrysopidae). Pesquisa Agropecuária Pernambucana, 23, 5.

Freitas, S. (2005). New species of Brazilian green lacewings genus Leucochrysa McLachlan, 1868 (Neuroptera: Chrysopidae). Annali de Museo civico di-Storia naturale di Ferrara, 8, 49-54.

Freitas, S., Penny, N. D. (2001). The green lacewings (Neuroptera: Chrysopidae) of Brazilian agro-ecosystems. Proceedings of the California Academy of Sciences, $52,245-395$.

González Olazo, E. V., Reguilón, C. (2008). Neuroptera. En: L. E. Claps, G. Debandi y S. Roig Juñent (eds.), Biodiversidad de Artrópodos Argentinos. Sociedad Entomológica Argentina 2, 235-248.

Mantoanelli, E., Albuquerque, G. S., Tauber, C. A., Tauber, M. J. (2006). Leucochrysa (Leucochrysa) varia (Neuroptera: Chrysopidae): larval descriptions, developmental rates, and adult color variation. Annals of the Entomological Society of America, 99, 7-18.

Mantoanelli, E., Albuquerque, G. S. (2007). Desenvolvimento e comportamento larval de Leucochrysa (Leucochrysa) varia (Schneider) (Neuroptera, Chrysopidae) em laboratório. Revista Brasileira de Zoologia, 24, 302-311. 
Mantoanelli, E., Tauber, C. A., Albuquerque, G. S., Tauber, M. J. (2011). Larvae of four Leucochrysa (Nodita) species (Neuroptera: Chrysopidae: Leucochrysini) from Brazil's Atlantic coast. Annals of the Entomological Society of America, 104, 1233-1259.

Oswald, J. D., Machado, R. J. (2018). Biodiversity of the Neuropterida (Insecta: Neuroptera, Megaloptera, and Raphidioptera). Insect Biodiversity: science and society, 2, 627-672.

Penny, N. D. (1978). Lista de Neuroptera, Megaloptera e Raphidioptera do México, América Central, ilhas Caraibas e América do Sul. Acta Amazonica, 7, 1-61.

Penny, N. D., Adams, P. A., Stange, L. A. (1997). Species catalog of the Neuroptera, Megaloptera, and Raphidioptera of America north of Mexico. Proceedings-California Academy Of Sciences, 50, 39-114.

Ramírez-Delgado, M., López-Arroyo, J. I., González-Hernández, A., \& Badii-Zabeh, M. H. (2007). Rasgos biológicos y poblacionales del depredador Ceraeochrysa sp. $n$ r. cincta (México) (Neuroptera: Chrysopidae). Acta zoológica mexicana, 23, 79-95.

Reguilón, C. (2010). Morfología de los estados inmaduros y ciclo biológico de Ungla binaria (Neuroptera: Chrysopidae). Acta zoológica lilloana, 54, 78-86.

Reguilón, C., Heredia, J. (2011). Morfología larval y datos biológicos de Leucochrysa (Nodita) vignisi (Neuroptera: Chrysopidae). Acta Zoológica Lilloana, 55, 147153.

Stehr, F. W. (1987). Techniques for collecting, rearing, preserving, and studying immature insects. Immature insects, 1, 7-18.

Solano, M., Guillén, C., Conejo, A. M. (2019). Entomología/Nota Técnica. Corbana, 45, 123-130.

Tauber, C. A. (2004). A systematic review of the genus Leucochrysa (Neuroptera: Chrysopidae) in the United States. Annals of the Entomological Society of America, 97, 1129-1158.

Tauber, C. A., Albuquerque, G. S., Tauber, M. J. (2008). A new species of Leucochrysa and a redescription of Leucochrysa (Nodita) clepsydra Banks (Neuroptera: Chrysopidae). Zootaxa, 1781, 1-19.

Tauber, C. A., Mantoanelli, E., Albuquerque, G. S., Reguilon, C., González Olazo, E., Tauber, M. J. (2011). A taxonomically significant polymorphism in Leucochrysa (Neuroptera: Chrysopidae): nomenclature, larval and adult descriptions, and biological notes. Zootaxa, 3130, 1-29.

Tauber, C. A., Sosa, F., Albuquerque, G. S., Tauber, M. J. (2013). Adults and larvae of two Leucochrysa (Leucochrysa) species (Neuroptera: Chrysopidae): descriptions, biological notes, and relationships. Zootaxa, 3750, 101-129. 